\title{
Lightning electromagnetic space distribution based the double exponential model
}

\author{
Ping $\mathrm{LU}^{1}$, Wei $\mathrm{HE}^{1^{*}}$, Jiajia $\mathrm{HE}^{2}$ \\ 1 School of Physics and Electronic Information, Yunnan Normal University, Kunming, China \\ 2 School of Physics and Electronic Information, Shanxi Normal University, Xi'an, China
}

Corresponding author: Wei HE, email: he99wei@aliyun.com

Keywords: Lightning; Electromagnetic field; Double exponential model

\begin{abstract}
Lightning, not only on the ground of buildings and electronic equipments but also for the aerospace, defense and military fields, equipment, facilities, has a great threat. In view of this situation, the deduced lightning electromagnetic field calculated expressions at any point in space is of great significance. Based on the assumption that the earth is a perfect conductor of the situation, and started from the Maxwell equations for the spatial distribution of lightning electromagnetic field calculation and analysis, we derived the electromagnetic field around the lightning channel by the lightning current expression. This expression reflects the lightning speed of the electromagnetic field and the lightning current comeback, lightning current size and field point height relationships between multiple factors. Finally, with the Matlab numerical simulation to get the electromagnetic field distribution space near the ground lightning, we would see it is coincide to the actual variation of lightning electromagnetic field and verified the feasibility of the model. Therefore, this method would provide a new theoretical basis for further study of lightning electromagnetic field.
\end{abstract}

\section{Introduction}

The lightning produces the powerful lightning current which caused the electromagnetic fields, the optical radiation, the shock wave and the thunder and so on physical effects. Strong currents generated by lightning would cause the strong variations electromagnetic radiation and electrostatic field, and it interferes with radio communications and a variety of remote devices work, which it becomes an important source of radio noise. On the other hand, the electromagnetic field generated by lightning is lightning detection of important information [1] [2]. Lightning-related research was started earlier, but until the 1990s, the research on lightning fight back electromagnetic field began to made significant progress by Rakov, etc. And Cooray has made negative lightning and positive lightning excitation electromagnetic field calculation model [3] [4]. Lupò obtained simple function of current by the research arbitrary shape and location of the lightning channel generated electromagnetic field [5]. Investigation on the lightning electromagnetic field began in the work of Uman et al in 1975. They assumed earth is the perfect conductor, and derived the express ions of the spatial electromagnetic field with lightning current of square wave form [6]. In this paper, with a double exponential function, and more useful results were obtained. Based on the previous, we derive the formula for lightning electromagnetic field, and we have some research on the spatial distribution of lightning electromagnetic field.

\section{Lightning channel model and the electromagnetic field}

Large numbers of observations have been proved that the lightning discharge channel is of the irregular and the arbitrary with a tilt and twisted branches and so on. At home and abroad on the lightning discharge channel modeling studies, it would be simplified and it is equivalent to a perpendicular to the ground conductor. According to the antenna theory research, we do not consider the branch and discharge waveform channels in the dissemination of deformation and other factors. The lightning discharge channel influences the electromagnetic field solving any field point in space due to lightning electromagnetic field analysis of the calculated expression. Suppose 
the current element is $i(\mathbf{R}, t) \mathbf{L}$, which $\mathrm{L}$ is the length of current element. According to the vector potential $\mathbf{A}(\mathbf{R}, t)$ and the Maxwell equations, we can calculate electric field $\vec{E}(\vec{R}, t)$ and magnetic field $\vec{B}(\vec{R}, t)$ generated the current element at any points.

Expression of the electric and magnetic fields were obtained by the vector potential $\mathbf{A}(\mathbf{R}, t)$ and scalar potential $\Phi(\mathbf{R}, \mathrm{t})$. Electric and magnetic fields can be obtained the expression.

$\mathbf{B}(r, \Phi, z, t)=\frac{\mu_{0} L}{4 \pi}\left[\frac{r}{c R^{2}} \frac{\partial i(t-R / c)}{\partial t}+\frac{r}{R^{3}} i(t-R / c)\right] \mathbf{a}_{\Phi}$

The lightning total electric field can be defined

$\mathbf{E}=E_{r} \mathbf{a}_{r}+E_{z} \mathbf{a}_{z}$

The $E_{r}$ component and $E_{z}$ component obtained expressions:

$E_{r}(r, \Phi, z, t)=\frac{L}{4 \pi \varepsilon_{0}}\left[\frac{3 r z}{R^{5}} \int_{0}^{t} i(\tau-R / c) d \tau+\frac{3 r z}{c R^{4}} i(t-R / c)+\frac{r z}{c^{2} R^{3}} \frac{\partial i(t-R / c)}{\partial t}\right]$

$E_{z}(r, \Phi, z, t)=\frac{L}{4 \pi \varepsilon_{0}}\left[\frac{2 z^{2}-r^{2}}{R^{5}} \int_{0}^{t} i(\tau-R / c) d \tau\right]+$

$\frac{L}{4 \pi \varepsilon_{0}}\left[\frac{2 z^{2}-r^{2}}{c R^{4}} i(t-R / c)-\frac{r^{2}}{c^{2} R^{3}} \frac{\partial i(t-R / c)}{\partial t}\right]$

\section{Lightning electromagnetic field numerical simulation and analysis}

In the actual lightning electromagnetic field of study, the surface $(z=0)$ is a specific field point, which people's production and life are in the ground field points. Similarly, for the actual measurements of lightning is carried out on the ground. The direct calculation of the space at any point electromagnetic field is difficult, because the formula (1) (4) (5) in a large number of the integral operation. Therefore, this chapter for ground lightning electromagnetic field point $(\mathrm{z}=0)$ at the numerical simulation, it verified the correctness of lightning electromagnetic field of theoretical calculations. Application of theoretical analysis and derivation formula calculates lightning current peak $\mathrm{Im}=200 \mathrm{kA}$, we set up the lightning channel height $\mathrm{H}=7.5 \mathrm{kM}$, the lightning discharge time 0 $\leqslant \mathrm{t} \leqslant 3 \mathrm{~s}$, the horizontal distance $0 \leqslant \mathrm{r} \leqslant 10 \mathrm{~km}$, current dipole scene point distance $\mathrm{R}$, the current element of length $\mathrm{L}$, the permeability $\mu_{0}=4 \pi \times 10^{7}$, the current comeback speed $\mathrm{c}=3 \times 10^{5} \mathrm{~m} / \mathrm{s}$, $\alpha=2.124 \times 10^{3}, \beta=2.456 \times 10^{5}$, the coefficient $(\mathrm{k}=1)$ determines the shape of the peak of the graph. In the Matlab simulation using the above data, the three-dimensional coordinates of the image of lightning electromagnetic fields are as follows:

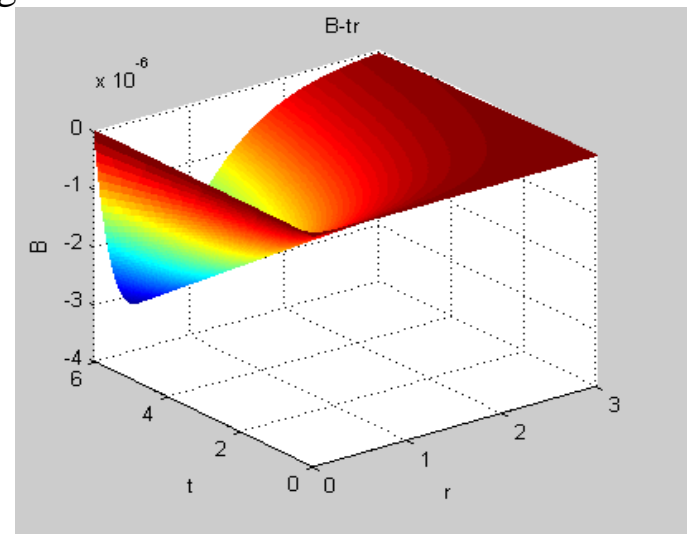

Figure 3.1 lightning electromagnetic field E-rz spatial distribution 


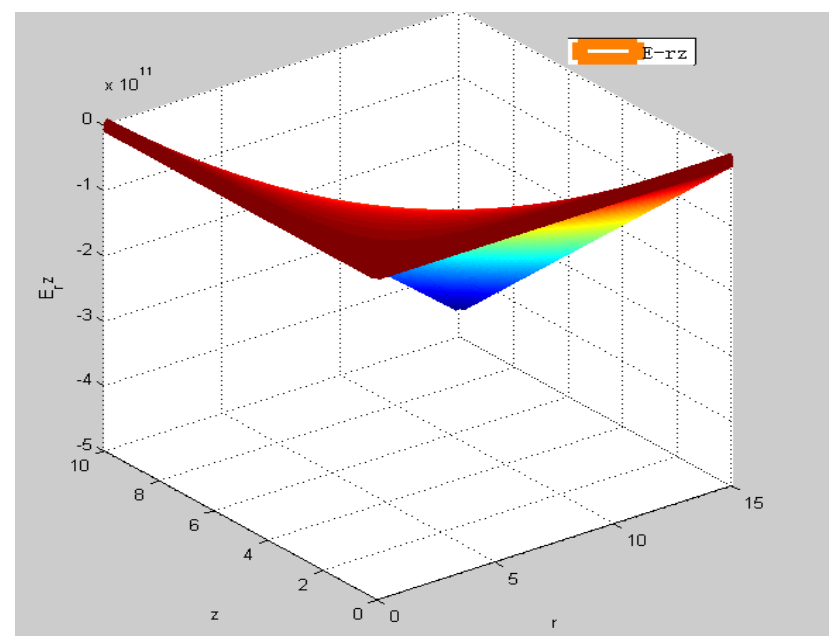

Figure 3.2 horizontal electric field B-tr spatial distribution

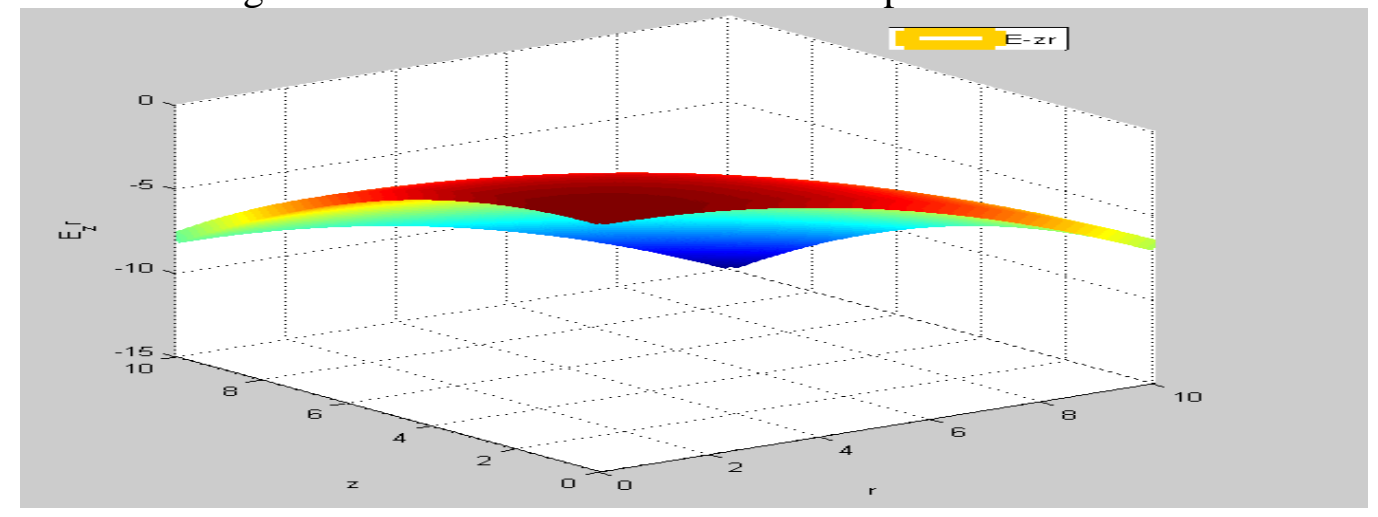

Figure 3.3 collimation of the electric field E-zr map distribution

For lightning electromagnetic field intensity distribution, from Figure 3.1 and Figure 3.3, $B_{\mathrm{tr}}$ and $E_{z r}$ of similar variation, when lightning current reaches a maximum, $B_{\mathrm{tr}}$ and $E_{z r}$ also peaked. And the same time, $B_{\mathrm{tr}}$ increases with the horizontal distance $\mathrm{r}$, extend the discharge time, and it would show a tendency to gradually decrease to zero. The $E_{z r}$ starting value is reached with the increase of $\mathrm{r}$ and $\mathrm{z}$ in the form of gradually decreasing trend. Figure 3.2 horizontal electric field $E_{r z}$ spatial distribution, just began to discharge, and the minimum level of the electric field with an increase in $\mathrm{r}$ and $\mathrm{z}$ when $E_{r z}$ was gradually increasing trend. Causing $E_{z r}$ and $E_{r z}$ appeared negative because lightning is negative lightning.

\section{Conclusion}

In this paper, the earth is under the condition of perfect conductor. The lightning electromagnetic field formula were derived, and from the results we can be drawn: (1) The inductive component caused by lightning current is proportional to the lightning current magnitude, while the radiation components of the ground horizontal magnetic field is varied with proportional to the lightning current to the derivative of time. (2) The electric field caused by the lightning current is divided into three parts: a) electrostatic field component, b) induction field component, c) electromagnetic radiation component. With a double exponential function of lightning current model we focus on the formula to be used the Matlab simulation. Finally we get the lightning electromagnetic field distribution space. The simulation results and the actual variation of lightning electromagnetic field is consistent, and it verify the feasibility of the model, and it can provide a theoretical basis for further study of lightning electromagnetic field. 


\section{Acknowledgment}

This project was supported by National Natural Science programs (50367001, 51267021) and Yunnan Province Science and Technology bureau program (2009ZC055M).

\section{References}

[1] Wei Ming. Lightning electromagnetic pulse and Protection [M] .Beijing: National Defense Industry Press, 2012, 1.11-66.

[2] Liuyou Ju and Wei He. Lightning discharge electromagnetic field and protect [M] .first edition .Kunming: Yunnan University Press, 2009.

[3] Valadimir A.Rakov, Martine A. Uman. Review and evaluation of lightning return stroke models including some aspects of their application[J].IEEE Transactions on Electromagnetic Compatibility,1998, 40(4):403- 426.

[4] Vernon Cooray. The modeling of positive return strokes in lightning flashes [J].Journal of Atmospheric and Solar- terrestrialphysics,2000,62:169- 187.

[5] Giovanni Lupò, Carlo Peterarca, Vincenzo Tucci, et al. EM fields generated by lightning channels with arbitrary location and slope[J].IEEE Transactions on Electromagnetic Compatibility,2000,42(1):39- 51.

[6] M. A. Uman, D. K. McLain, E. P. Krider. The electromagnetic radiation from a finite antenna[J]. Amer. J. Phys. 1975, 43(6) : 33-38. 